\title{
Manejo anestésico y de vía aérea en el paciente con osteosarcoma mandibular de alto grado de crecimiento acelerado
}

\author{
Anesthetic and airway management in patients with \\ high-grade, fast-growing jaw osteosarcoma
}

\author{
Gloria Beatriz Fábregas Popoca, ${ }^{*}$ Karen Itzel Escamilla Godínez ${ }^{\ddagger}$
}

Citar como: Fábregas PGB, Escamilla GKI. Manejo anestésico y de vía aérea en el paciente con osteosarcoma mandibular de alto grado de crecimiento acelerado. An Med ABC. 2021; 66 (3): 210-213. https://dx.doi.org/10.35366/101669

\section{RESUMEN}

La dificultad en el manejo de las vías respiratorias en los pacientes con cáncer de mandíbula se debe principalmente a los cambios anatómicos y fisiológicos, así como a la radioterapia y la quimioterapia adyuvante. El manejo de la vía aérea se considera un desafío en estos pacientes con respecto a los problemas inherentes de la vía aérea difícil, debido a la masa. Se describe el caso de una paciente de 19 años, previamente sana, con diagnóstico de osteosarcoma grado 3 de crecimiento rápido, programada para mandibulectomía, traqueotomía y gastrostomía y el manejo anestésico enfocado en la vía aérea.

Palabras clave: Vía aérea difícil, tumor maxilar, osteosarcoma.

\section{INTRODUCCIÓN}

El osteosarcoma es el tumor óseo maligno más común (excluidos los tumores de la médula ósea), y los osteosarcomas mandibulares y maxilares representan sólo $6 \%$ de todos los osteosarcomas. ${ }^{1}$

Hasta ahora no se han descrito diferencias patohistológicas, radiológicas o inmunohistoquímicas

\begin{abstract}
Difficulty in airway management in patients with jaw cancer is mainly due to anatomical and physiological changes, as well as radiotherapy and adjuvant chemotherapy. Airway management is considered challenging in these patients with respect to the inherent problems of difficult airway due to mass. The case of a 19-year-old female is described, previously healthy with a diagnosis of fast-growing grade 3 osteosarcoma, scheduled for mandibulectomy, tracheostomy and gastrostomy and the anesthetic management that was offered, focused on the management of the airway.
\end{abstract}

Keywords: Difficult airway, maxillary tumor, osteosarcoma.

particulares entre el osteosarcoma de mandíbula y el de hueso periférico. Sin embargo, el de mandíbula muestra varias características clínicas distintas, los pacientes con osteosarcoma de mandíbula son, en promedio, aproximadamente dos décadas mayores y tienden a tener un riesgo menor de diseminación hematológica, lo que se asocia con tasas de supervivencia más altas. ${ }^{2}$ El tratamiento se basa y comprende

\footnotetext{
* Médica anestesióloga. Coordinadora del Servicio de Anestesiología en Cirugía Oncológica.

‡ Médico residente de tercer año en Anestesiología.
}

Hospital General de México «Eduardo Liceaga».
Recibido: 27/05/2020. Aceptado: 04/02/2021.

\section{Correspondencia:}

Dra. Gloria Beatriz Fábregas Popoca

E-mail: drafabregasgloria@gmail.com 
de quimioterapia neoadyuvante, seguida de resección quirúrgica y quimioterapia adyuvante. ${ }^{3}$

El procedimiento quirúrgico sigue siendo complicado en localizaciones faciales porque es difícil obtener márgenes quirúrgicos libres, lo que conduce a alteraciones funcionales y estéticas. ${ }^{4}$

\section{REPORTE DE CASO}

Paciente femenino de 19 años de edad con diagnóstico de sarcoma mandibular, programada para mandibulectomía, traqueotomía y gastrostomía.

El único antecedente de importancia es asma de cinco años de diagnóstico sin tratamiento actualmente, siendo su última crisis hace cuatro años.

Inició su padecimiento en noviembre de 2020 con parestesias y disestesias en región mandibular, así como aumento de volumen. Acude a facultativo, quien dio tratamiento antibiótico sin mejoría, por lo que se envió a toma de biopsia el 21 de marzo de 2021, donde se da diagnóstico de osteosarcoma mandibular grado 3 de predominio izquierdo, por lo que se envió al Área de Tumores de Cabeza y Cuello del Hospital General de México «Eduardo Liceaga».

Tomografía de cabeza y cuello del 12 de abril reportó una lesión ósea que afecta ambas ramas mandibulares con reacción perióstica en sol naciente, con extensión a tejidos blandos de la región oral de dimensiones $6 \times 6.5 \mathrm{~cm}$. El 15 de abril de 2021 se refirió al Área de Oncología Médica del mismo hospital para tratamiento adyuvante previo a evento quirúrgico. El 28 de abril es valorada por este servicio y se decidió iniciar quimioterapia con doxirrubicina $39 \mathrm{mg}$ IV más cisplatino $159 \mathrm{mg}$ IV, se dan dos ciclos de quimioterapia (12 de abril y 15 de mayo) sin aparente mejoría y presentando anemia severa que requirió de múltiples trasfusiones sanguíneas, por lo que se decide programar evento quirúrgico el día 10 de agosto de 2021. El 21 de julio de 2021 se ingresó a este hospital para inicio de protocolo prequirúrgico y optimización de la paciente (Figura 1).

En la valoración preoperatoria por anestesiología del día 18 de julio de 2021, se encontró paciente consciente, orientada, con palidez de tegumentos, adecuada hidratación, se observó tumor mandibular con dimensiones mayores a la reportadas en la tomografía computarizada, con limitación de la apertura oral, vía área difícil, un Mallampati IV, Patil-Aldrete de I y DEM mayor a $6.5 \mathrm{~cm}$.

Laboratorios preoperatorios: hemoglobina 13.1, hematocrito 40.5 , leucocitos de 7.6 plaquetas de
265000, TP 12.4, TPT 27.4 e INR 1.1. Se solicitó tener disponible catéter venoso central, el cual fue colocado el 21 de julio de 2021, tres frascos de albúmina y tres concentrados eritrocitarios con tres plasmas frescos congelados.

Se le explicó la necesidad de intubación y/o traqueotomía despierta, se le explican beneficios, probables complicaciones y riesgos, por lo que aceptó el procedimiento.

Ingresó a sala, se colocó monitorización invasiva, con PANI, ECG, pulsioximetría, capnografía, capnometría, presión arterial invasiva, BIS, uresis horaria y sistema VolumeView TM de Edwards para monitoreo hemodinámico. Signos vitales al ingreso: tensión arterial 134/105 mmHg, frecuencia cardiaca de $98 \mathrm{lpm}$, frecuencia respiratoria de $16 \mathrm{rpm}, \mathrm{SaO}_{2}$ de $95 \%$. Técnica anestésica: sedación para traqueotomía en paciente despierto, AGB para los procedimientos restantes.

Se inició sedación con dexmedetomidina $0.5 \mu \mathrm{g} /$ $\mathrm{kg} / \mathrm{min}$ y lidocaína a $25 \mu \mathrm{g} / \mathrm{kg} 7 \mathrm{~min}$ en perfusión intravenosa durante 20 minutos, midazolam bolos fraccionados con un total de $2 \mathrm{mg}$, fentanil en bolos fraccionados con un total de $200 \mu \mathrm{g}$. Se realizó traqueotomía, donde se colocó inicialmente sonda orotraqueal no. 6.5 con $3 \mathrm{~cm}^{3}$ de neumotaponamiento (Figura 2).

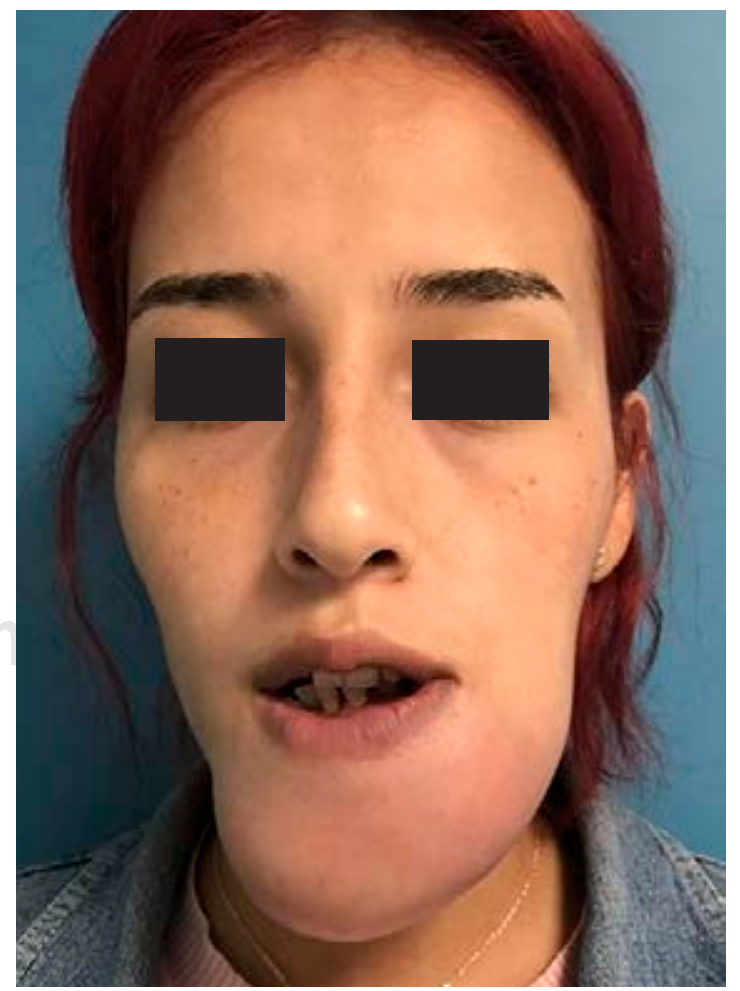

Figura 1: Paciente en su primera consulta en abril 2021. 


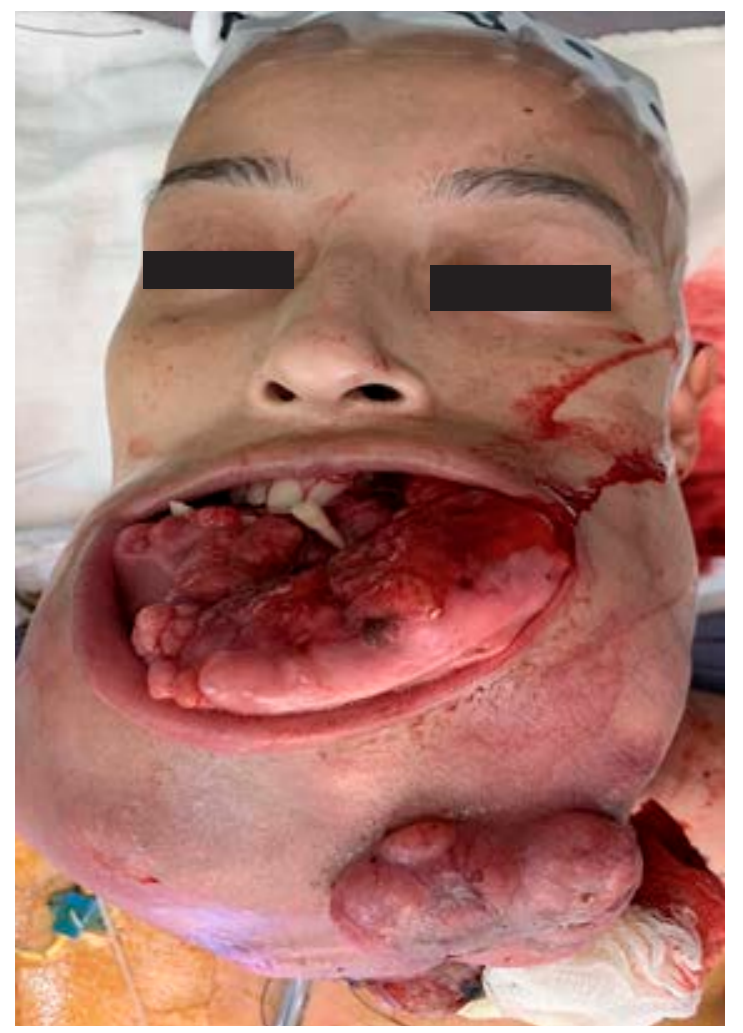

Figura 2: Paciente el día de la cirugía, posterior a traqueotomía.

Ventilación mecánica modo controlado por volumen, VC: $350 \mathrm{~mL}$, FR 12-18 rpm, I:E i:2, PEEP 5 $\mathrm{cmH}_{2} \mathrm{O}, \mathrm{FiO}_{2} 60 \%$. Se colocó línea arterial radial derecha No. 22, donde se posicionó el sensor Edwards para línea arterial y monitoreo hemodinámico, y se instauró vía periférica No. 18 en dorso de mano izquierda y dorso pie izquierdo. Mantenimiento: sevoflorano a 1.6 vol. \% para CAM de 0.6 y Bis entre 40 y 60, perfusiones IV: fentanil dosis total $970 \mu \mathrm{g}$, dexmedetomidina dosis total $95 \mu \mathrm{g}$, lidocaína dosis total 270 mg. Gasometría inicial con $\mathrm{pH}$ : 7.36, $\mathrm{PaO}_{2}: 109$, $\mathrm{PaCO}_{2}: 35,7, \mathrm{HCO}_{3}: 20.3, \mathrm{BE}:-5.0, \mathrm{Hb}: 5.8 \mathrm{Hto}: 17$ glucosa: 212 Lactato: 2.39 .

Transanestésico: ingresó paciente con taquicardia e hipertensa, lo cual se mantuvo hasta la resección de la pieza quirúrgica, con datos de resistencias vasculares y volumen sistólico izquierdo por debajo de límites normales, posterior a la resección disminuye TAM a menos de $60 \mathrm{mmHg}$, por lo que se inició vasopresor a dosis respuesta $(0.02-0.22 \mu \mathrm{g} / \mathrm{kg} / \mathrm{min})$ para mantener TAM mayor a $65 \mathrm{mmHg}$ (Figura 3).

Se trasfundieron tres concentrados eritrocitarios con un volumen total de $860 \mathrm{~mL}+2$ PFC con un volumen total de $190 \mathrm{~mL}$. Se retiró tubo endotraqueal y se colocó sonda de traqueotomía No. 7 sin incidentes. Gasometría al término con $\mathrm{pH}: 7.42, \mathrm{PaO}_{2}: 181$, $\mathrm{PaCO}_{2}: 35.9, \mathrm{HCO}_{3}: 23.5, \mathrm{BE}:-1.2, \mathrm{Hb}: 10.7$, Hto: 31 , Glucosa: 55, Lactato: 2.43. Sangrado total de $750 \mathrm{~mL}$.

Egresó a la Unidad de Cuidados Postanestésicos con ventilación espontánea y hemodinámicamente estable; se mantuvo en vigilancia por 90 minutos con una adecuada recuperación, por lo que se decidió su alta a pabellón.

\section{DISCUSIÓN}

La dificultad en el manejo de la vía aérea en los pacientes con cáncer de mandíbula se debe principalmente a los cambios anatómicos y fisiológicos, así


Figura 3: A) Paciente al término de la cirugía. B) Tumor resecado donde se puede apreciar la destrucción de piezas dentales. 
como a la radioterapia y la quimioterapia adyuvante. La incidencia de vía aérea difícil en el cáncer de cabeza y cuello es mayor que en la población general. ${ }^{5}$ El manejo de la vía aérea se considera un desafío en estos pacientes con respecto a los problemas inherentes de la vía aérea difícil, debido a la masa, retroceso de la mandíbula, apertura de la boca y movimiento del cuello restringidos o, también, a las comorbilidades asociadas. La evaluación de la vía aérea debe incluir una valoración de la dificultad de la intubación y la viabilidad de planes de rescate adecuados para lograr la oxigenación. . $^{5-7}$

El principal objetivo intraoperatorio es la elección adecuada de la técnica de la vía aérea y el dispositivo de la vía aérea adaptados al paciente y a la técnica quirúrgica. Otros objetivos son la provisión de un manejo experto de la vía aérea, un plano continuo de anestesia, un campo quirúrgico despejado e inmovilizado, una recuperación suave y rápida de la anestesia. ${ }^{8}$

Aunque la intubación nasotraqueal con fibra óptica es la técnica más preferida en el manejo de una vía aérea difícil. La frecuencia y la incidencia de una traqueotomía se pueden controlar mediante una planificación preoperatoria adecuada. ${ }^{7}$ La necesidad de una monitorización avanzada o invasiva del gasto cardiaco está determinada por las comorbilidades del paciente, así como por la naturaleza y extensión de la cirugía. ${ }^{9}$ El mantenimiento de la anestesia se puede obtener con anestesia intravenosa total o anestésicos por inhalación o una combinación de anestésico por inhalación con infusión intravenosa de un anestésico de acción corta y se basa en los factores del paciente, las necesidades quirúrgicas y el potencial de ventilación comprometida. ${ }^{8,9}$

\section{CONCLUSIÓN}

La cirugía de osteosarcoma mandibular plantea mayores desafíos para el anestesiólogo; se debe optimizar las comorbilidades del paciente en el preoperatorio, compartir la vía aérea, implementar diferentes técnicas de anestesia y vía aérea específicas para la cirugía y manejar las complicaciones postoperatorias de la vía aérea. Estos pacientes presentan la vía aé- rea difícil debido a la patología que impide la anatomía de la vía aérea. Por lo tanto, la planificación preoperatoria requiere una anamnesis y un examen físico minuciosos con especial atención a la evaluación de la vía aérea. Los pacientes de alto riesgo deben identificarse preoperatoriamente y optimizarse médicamente antes de la cirugía. Un enfoque multidisciplinario y con experiencia debe fomentar la anestesia y el manejo avanzado de la vía aérea, ya que disminuye las complicaciones de la misma y mejora los resultados quirúrgicos.

\section{REFERENCIAS}

1. ElKordy MA, ElBaradie TS, ElSebai HI, KhairAlla SM, Amin AAE. Osteosarcoma of the jaw: Challenges in the diagnosis and treatment. J Egypt Natl Canc Inst. 2018; 30 (1): 7-11. doi: 10.1016/j.jnci.2018.02.001.

2. Eder-Czembirek C, Moser D, Holawe S, Brodowicz T, Ries J, Sulzbacher I, Selzer E. Osteosarcoma of the jaw - experience at the Medical University Vienna and comparative study with international tumor registries. Clinics (Sao Paulo). 2019; 74: e701. doi: 10.6061/clinics/2019/e701.

3. Crenn V, Biteau K, Amiaud J, Dumars C, Guiho R, Vidal $\mathrm{L}$ et al. Bone microenvironment has an influence on the histological response of osteosarcoma to chemotherapy: retrospective analysis and preclinical modeling. Am J Cancer Res. 2017; 7 (11): 2333-2349.

4. Bertin H, Guilho R, Brion R, Amiaud J, Battaglia S, Moreau A et al. Jaw osteosarcoma models in mice: first description. Journal of Translational Medicine. 2019; 17 (1): doi: 10.1186/ s12967-019-1807-5.

5. Ahmed-Nusrath A. Anesthesia for head and neck cancer surgery, BJA Education. 2017; 17 (12): 383-389. Available in: https://doi.org/10.1093/bjaed/mkx028

6. Charters P, Ahmad I, Patel A, Russell S. Anaesthesia for head and neck surgery: united kingdom national multidisciplinary guidelines. J Laryngol Otol. 2016; 130 (S2): S23-S27. doi: 10.1017/S0022215116000384.

7. Nagarkar R, Kokane G, Wagh A, Kulkarni N, Roy S, Tandale R et al. Airway management techniques in head and neck cancer surgeries: a retrospective analysis. Oral and Maxillofacial Surgery. 2019; 23 (3): 311-315. doi: 10.1007/s10006-01900782-1.

8. Coyle MJ, Main B, Hughes C, Craven R, Alexander R, Porter $\mathrm{G}$ et al. Enhanced recovery after surgery (ERAS) for head and neck oncology patients. Clin Otolaryngol. 2016; 41 (2): 118-126.

9. Jaffar KM, Tageldin T, Waqas FM, Qasim KO, Shahid U, El-Fatih ARM et al. Principles of anesthesia and airway management in head and neck surgery. Surgical management of head and neck pathologies. Published. 2020; Available in: https://doi.org/10.5772/intechopen.95029 